\title{
Proton puzzles
}

Over a century after its discovery, the proton still keeps physicists busy understanding its basic properties, but a new generation of experiments may help finally nail down its radius, stability and the origin of its spin.

Only about $5 \%$ of our Universe is ordinary matter: stuff made of particles described by the standard model, that physicists have a reasonably good understanding of. But even for some of these particles unanswered questions linger. Every schoolchild knows that atoms are made of protons, neutrons and electrons and young science enthusiasts might even know that, unlike electrons which are elementary particles, protons are made of quarks. What is less known is that physicists have been scratching their heads for decades trying to figure out some basic properties of the proton.

How big is the proton? Since the 1950s the proton charge radius has been measured using either elastic electron-proton scattering or hydrogen atomic spectroscopy. But in 2010 a new measurement using muonic hydrogen spectroscopy disagreed with the accepted value. The fact that "the proton seen by muons thus appeared smaller than that seen by electrons" puzzled the physics community and it took almost a decade to be more-or-less understood. More recent electron scattering and ordinary hydrogen spectroscopy measurements agree with the results from muonic atom spectroscopy, but this is not yet the final resolution of the proton radius puzzle. It may be that the tension between the different measurements of the proton charge radius is the result of poorly understood systematic errors in these methods. A new generation of experiments discussed in a recently published Review ${ }^{1}$ by Jean-Philippe Karr, Dominique March and Eric Voutier is expected to settle this question.

Where does the proton spin come from? The proton is a spin- $1 / 2$ particle. It would seem reasonable to assume that its spin comes from the simple arithmetic of three component quarks (spin-1/2) - two up and one down. But in 1987 the European Muon Collaboration at CERN measured the individual quark spin contributions using polarized deep-inelastic scattering of electrons or muons off a polarized proton target, and found that the quarks only account for a small fraction the total proton spin. Over the past 30 years two main approaches have been developed to study the additional contributions to the proton spin. These methods, discussed in a Review ${ }^{2}$ by Xiangdong Ji, Feng Yuan and
Yong Zhao in this issue, together with advances in spin ab initio calculations in lattice quantum chromodynamics, have revealed much about the proton spin structure. The final pieces of the puzzle are expected to come from experiments at the Electron-Ion Collider $(\mathrm{EIC})^{2}$. The construction of the EIC has just started and in a Comment in this issue, James Yeck explains the challenges in delivering big science projects and EIC in particular.

Does the proton decay? The proton could decay into a positron and a pion and if so the predictions of some beyond standard model grand unified theories would be confirmed. Since the 1980s underground experiments have been looking for signs of such decay. No proton decay has yet been spotted, but one of the experiments, the Kamioka Nucleon Decay Experiment, or KamiokaNDE, detected neutrinos from the supernova SN1987a instead. Its successor, the Super-Kamiokande, set the best current lower-limit for proton lifetime: $1.6 \times 10^{34}$ years. Some grand unified theories predict proton lifetimes up to around $10^{36}$ years so there is still room for surprises. Upcoming neutrino experiments also have proton decay measurements planned and are expected to push the limits on the proton lifetime. These measurements will take place at Deep Underground Neutrino Experiment (DUNE) currently under construction, Jiangmen Underground Neutrino Observatory (JUNO) which is almost completed and Hyper-Kamiokande whose construction received the green light last year.

A century after Ernest Rutherford discovered the proton, it is both a textbook particle and a topic of active research. This is a nice illustration of what science is about: not only exploring the unknown, but also carefully scrutinizing and continuously updating what is considered to be known.

1. Karr, Jean-Philippe, Marchand, Dominique $\&$ Voutier, Eric. The proton size. Nat. Rev. Phys. 2, 601-614 (2020).

2. Ji, Xiangdong, Yuan, Feng $\&$ Zhao, Yong. What we know and what we don't know about the proton spin after 30 years. Nat. Rev. Phys. https://doi.org/10.1038/s42254-020-00248-4 (2020).

\footnotetext{
RELATED LINKS

The proton laid bare, CERN Courier (2019) https://cerncourier.com/a/the-
} proton-laid-bare/ 